\title{
On the Milnor fiber of a real map-gem
}

\author{
Nicolas DUTERTRE
}

(Received July 14, 2000)

\begin{abstract}
We give an algebraic formula for a topological invariant of real analytic singularities. We deduce from this formula a new proof of the topological invariance of the Milnor number mod 2.
\end{abstract}

Key words: real Milnor fiber, local algebra, Euler characteristic.

\section{Introduction}

Let $f=\left(f_{1}, \ldots, f_{k}\right):\left(\mathbf{K}^{n}, 0\right) \rightarrow\left(\mathbf{K}^{k}, 0\right)$, with $1 \leq k<n$ and $\mathbf{K}=\mathbf{C}$ or $\mathbf{K}=\mathbf{R}$, be an analytic germ defined in a neighborhood of the origin. We are interested in computing topological invariants associated to the mapping $f$.

Let $B_{\varepsilon} \subset \mathbf{K}^{n}$ be a small closed ball centered at the origin and let $\delta \in$ $\mathbf{K}^{k}$ be a small regular value of $f$. The Milnor fiber of $f$ is $f^{-1}(\delta) \cap B_{\varepsilon}$. If $k=1, \mathbf{K}=\mathbf{C}$ and $f$ has an isolated critical point at 0 , Milnor [Mi2] proved that $f^{-1}(\delta) \cap B_{\varepsilon}$ has the homotopy type of a bouquet of $\mu$ spheres of dimension $n-1$. This number of spheres is called the Milnor number of $f$, and according to Milnor [Mi2] and Palamodov [Pa],

$$
\mu=\operatorname{dim}_{\mathbf{C}} \frac{\mathcal{O}_{\mathbf{C}^{n}, 0}}{\left(\frac{\partial f}{\partial x_{1}}, \ldots, \frac{\partial f}{\partial x_{n}}\right)},
$$

where $\mathcal{O}_{\mathbf{C}^{n}, 0}$ is the ring of germs of analytic functions defined at the origin.

This result was extended to the case $1<k<n$ by Hamm, who proved that the Milnor fiber has the homotopy type of a bouquet of $\mu$ spheres of dimension $n-k$, and by Lê [Le] and Greuel [Gr] who obtained the following formula

$$
\mu\left(f^{\prime}\right)+\mu(f)=\operatorname{dim}_{\mathbf{C}} \mathcal{O}_{\mathbf{C}^{n}, 0} / I,
$$

where $f^{\prime}=\left(f_{1}, \ldots, f_{k-1}\right)$ and $I$ is the ideal generated by $f_{1}, \ldots, f_{k-1}$ and all $k \times k$ minors $\frac{\partial\left(f_{1}, \ldots, f_{k}\right)}{\partial\left(x_{i_{1}}, \ldots, x_{i_{k}}\right)}$. 
In the real case, it is difficult to give such precise information about the topology of the Milnor fiber. Nevertheless, it is possible to compute some Euler characteristics. For example, if $k=1$ and $f$ has an isolated critical point at the origin, the Khimshiasvili's formula $([\mathrm{Ar}],[\mathrm{Fu} 2],[\mathrm{Kh}]$, [Wa]) states that

$$
\chi\left(f^{-1}(\delta) \cap B_{\varepsilon}\right)=1-\operatorname{sign}(-\delta)^{n} \operatorname{deg}_{0} \nabla f,
$$

where $\operatorname{deg}_{0} \nabla f$ is the topological degree of the gradient of $f$ at the origin. This formula can be viewed as a real version of the formula (1) above. The aim of this paper is to give a real version of the Lê-Greuel formula, i.e. the formula (2) above.

We first introduce the situation. Let $f=\left(f_{1}, \ldots, f_{k}\right):\left(\mathbf{R}^{n}, 0\right) \rightarrow$ $\left(\mathbf{R}^{k}, 0\right)$, with $n>k$, be an analytic map and let $g:\left(\mathbf{R}^{n}, 0\right) \rightarrow(\mathbf{R}, 0)$ be an analytic function. Let $I$ be the ideal generated by $f_{1}, \ldots, f_{k}$ and all $(k+1) \times(k+1)$ minors $\frac{\partial\left(g, f_{1}, \ldots, f_{k}\right)}{\partial\left(x_{i_{1}}, \ldots, x_{i}\right)}$ in $\mathcal{O}_{\mathbf{R}^{n}, 0}$, the ring of germs of analytic functions at the origin. Let $\delta \in \mathbf{R}^{k}$ be a regular value of $f$ and let $\alpha \in \mathbf{R}$ such that $|\alpha| \ll|\delta|$ and $(\delta, \alpha)$ is a regular value of $(f, g)$. Assuming that $\operatorname{dim}_{\mathbf{R}} \mathcal{O}_{\mathbf{R}^{n}, 0} / I<+\infty$, we will prove the following result (see Theorem 3.8)

$$
\begin{aligned}
& \chi\left(f^{-1}(\delta) \cap\{g \geq \alpha\} \cap B_{\varepsilon}\right)+\chi\left(f^{-1}(\delta) \cap\{g \leq \alpha\} \cap B_{\varepsilon}\right) \\
& \quad \equiv \operatorname{dim}_{\mathbf{R}} \frac{\mathcal{O}_{\mathbf{R}^{n}, 0}}{I} \bmod 2 .
\end{aligned}
$$

This theorem generalizes the case $g=x_{1}^{2}+\cdots+x_{n}^{2}$ which was already proved by Dudzinski et al. in [DLNS], using fixed point theory and the Lê-Greuel formula. It is also a mod 2 generalization of the formulas for counting the number of branches of a one-dimensional semi-analytic set given by Aoki et al. ([AFN1], [AFN2], [AFS] $)$ and by Szafraniec ([Sz1]).

Now let us consider the complexification $f_{\mathbf{C}}:\left(\mathbf{C}^{n}, 0\right) \rightarrow\left(\mathbf{C}^{k}, 0\right)$ of $f$. Let $\mu(f)$ be the Milnor number of $f_{\mathbf{C}}$. Let $L(f)$ be the link of $f$ and let $\psi(f)$ be the semi-characteristic of $L(f)$. We recall that the semi-characteristic is defined to be half the sum of the mod 2 Betti numbers. C.T.C Wall ([Wa] $)$ showed that

$$
\psi(f) \equiv 1+\mu(f) \bmod 2 .
$$

As a corollary, one gets that $\mu(f) \bmod 2$ is a topological invariant of $f$. Wall's proof is straightforward for the case $k=1$. The case of arbitrary $k$ is more complicated; Wall gives a sophisticated topological argument using 
spectral sequence from fixed point theory. At the end of his paper, he asks if there is a proof of this result more like the case for $k=1$. Using Theorem 3.8, we will supply a proof of this type (see Theorem 4.5).

The paper is organized as follows: in Section 2, we recall some facts about Morse theory for manifolds with boundary; Section 3 is devoted to the proof of our main formula; in Section 4, we give a new proof of the topological invariance of the Milnor number mod 2. The author is very grateful to Karim Bekka for his helpful remarks and comments.

\section{Morse theory for manifolds with boundary}

We recall the results of Morse theory for manifolds with boundary. Our reference is [HL] where the results are given for a $C^{\infty}$ manifold $M$ with boundary $\partial M$. For simplicity we will present the results for manifolds with boundary of type $M \cap\{g * 0\}, * \in\{\geq, \leq\}$, where $M$ is a $C^{\infty}$ manifold and $g: M \rightarrow \mathbf{R}$ a $C^{\infty}$ function such that $M \cap g^{-1}(0)$ is smooth. In fact this is the case we need in the following sections.

Let $M$ be a $C^{\infty}$ manifold of dimension $n$. Let $g: M \rightarrow \mathbf{R}$ be a $C^{\infty}$ function such that $\nabla g(x) \neq 0$ for all $x \in g^{-1}(0)$. This implies that $M \cap$ $g^{-1}(0)$ is a smooth manifold of dimension $n-1$ and that $M \cap\{g \geq 0\}$ and $M \cap\{g \leq 0\}$ are smooth manifolds with boundary. Let $f: M \rightarrow \mathbf{R}$ be a smooth function. A critical point of $f_{\mid M \cap\{g \geq 0\}}$ (resp. $f_{\mid M \cap\{g \leq 0\}}$ ) is a critical point of $f_{\mid M \cap\{g>0\}}$ (resp. $f_{\mid M \cap\{g<0\}}$ ) or a critical point of $f_{\mid M \cap g^{-1}(0)}$.

Definition 2.1 Let $q \in M \cap g^{-1}(0)$. We say that $q$ is a correct critical point of $f_{\mid M \cap\{g \geq 0\}}$ (resp. $f_{\mid M \cap\{g \leq 0\}}$ ) if $q$ is a critical point of $f_{\mid M \cap g^{-1}(0)}$ and $q$ is not a critical point of $f_{\mid M}$.

We say that $q$ is a correct non-degenerate critical point of $f_{\mid M \cap\{g \geq 0\}}$ (resp. $f_{\mid M \cap\{g \leq 0\}}$ ) if $q$ is a correct critical point of $f_{\mid M \cap\{g \geq 0\}}$ (resp. $f_{\mid M \cap\{g \leq 0\}}$ ) and $q$ is a non-degenerate critical point of $f_{\mid M \cap g^{-1}(0)}$.

If $q$ is a correct critical point of $f_{\mid M \cap\{g \geq 0\}}$ (resp. $\left.f_{\mid M \cap\{g \leq 0\}}\right)$ then $\nabla f(q) \neq$ $\overrightarrow{0}, \nabla f(q)$ and $\nabla g(q)$ are colinear and there is $\tau(q) \in \mathbf{R}^{*}$ with $\nabla f(q)=\tau(q)$. $\nabla g(q)$.

Definition 2.2 If $q$ is a correct critical point of $f_{\mid M \cap\{g \geq 0\}}$ then

- $\nabla f(q)$ points inwards if and only if $\tau(q)>0$,

- $\nabla f(q)$ points outwards if and only if $\tau(q)<0$. 
If $q$ is a correct critical point of $f_{\mid M \cap\{g \leq 0\}}$ then

- $\nabla f(q)$ points inwards if and only if $\tau(q)<0$,

- $\nabla f(q)$ points outwards if and only if $\tau(q)>0$.

Definition 2.3 A $C^{\infty}$ function $f: M \cap\{g \geq 0\} \rightarrow \mathbf{R}$ (resp. $M \cap\{g \leq$ $0\} \rightarrow \mathbf{R})$ is a correct function if all critical points of $f_{\mid M \cap g^{-1}(0)}$ are correct. A $C^{\infty}$ function $f: M \cap\{g \geq 0\} \rightarrow \mathbf{R}$ (resp. $M \cap\{g \leq 0\} \rightarrow \mathbf{R}$ ) is a Morse correct function if $f_{\mid M \cap\{g>0\}}$ (resp. $f_{\mid M \cap\{g<0\}}$ ) admits only non-degenerate critical points and if $f$ admits only non-degenerate correct critical points.

Proposition 2.4 For all $C^{\infty}$ manifold $M$ and for all function $g: M \rightarrow \mathbf{R}$ such that $\nabla g(x) \neq 0$ for all $x \in g^{-1}(0)$, the set of $C^{\infty}$ functions $f: M \rightarrow$ $\mathbf{R}$ such that $f_{\mid M \cap\{g \geq 0\}}$ and $f_{\mid M \cap\{g \leq 0\}}$ are Morse correct functions is dense in $C^{\infty}(M, \mathbf{R})$.

We will denote $\chi(M \cap\{g * 0\} \cap\{f ? 0\})$, where $*, ? \in\{\leq,=, \geq\}$, by $\chi_{*,}$ ? and we will use the following result:

Theorem 2.5 Let $M$ be a $C^{\infty}$ compact manifold of dimension $n$ and let $g: M \rightarrow \mathbf{R}$ be a $C^{\infty}$ function such that $\nabla g(x) \neq 0$ for all $x \in g^{-1}(0)$. Let $f: M \rightarrow \mathbf{R}$ be a $C^{\infty}$ function such that $f_{\mid M \cap\{g \geq 0\}}$ and $f_{\mid M \cap\{g \leq 0\}}$ are Morse correct. Let $\left\{p_{i}\right\}$ be the set of critical points of $f_{\mid M}$ and $\left\{\lambda_{i}\right\}$ be the set of their respective indices. Let $\left\{q_{j}\right\}$ be the set of critical points of $f_{\mid M \cap g^{-1}(0)}$ and $\left\{\mu_{j}\right\}$ be the set of their respective indices. Then we have

$$
\begin{aligned}
& \chi_{\geq, \geq}-\chi_{\geq,=}=\sum_{\substack{i / f\left(p_{i}\right)>0 \\
g\left(p_{i}\right)>0}}(-1)^{\lambda_{i}}+\sum_{\substack{j / f\left(q_{j}\right)>0 \\
\tau\left(q_{j}\right)>0}}(-1)^{\mu_{j}}, \\
& \chi_{\geq, \leq-} \chi_{\geq,=}=(-1)^{n} \sum_{\substack{i / f\left(p_{i}\right)<0 \\
g\left(p_{i}\right)>0}}(-1)^{\lambda_{i}}+(-1)^{n-1} \sum_{\substack{j / f\left(q_{j}\right)<0 \\
\tau\left(q_{j}\right)<0}}(-1)^{\mu_{j}},
\end{aligned}
$$

and

$$
\begin{aligned}
& \chi_{\leq, \geq}-\chi_{\leq,=}=\sum_{\substack{i / f\left(p_{i}\right)>0 \\
g\left(p_{i}\right)<0}}(-1)^{\lambda_{i}}+\sum_{\substack{j / f\left(q_{j}\right)>0 \\
\tau\left(q_{j}\right)<0}}(-1)^{\mu_{j}}, \\
& \chi_{\leq, \leq}-\chi_{\leq,=}=(-1)^{n} \sum_{\substack{i / f\left(p_{i}\right)<0 \\
g\left(p_{i}\right)<0}}(-1)^{\lambda_{i}}+(-1)^{n-1} \sum_{\substack{j / f\left(q_{j}\right)<0 \\
\tau\left(q_{j}\right)>0}}(-1)^{\mu_{j}} .
\end{aligned}
$$




\section{The main formula}

In this section, we prove the result announced in the introduction for a real map germ. We recall that $f=\left(f_{1}, \ldots, f_{k}\right):\left(\mathbf{R}^{n}, 0\right) \rightarrow\left(\mathbf{R}^{k}, 0\right)$ is an analytic germ, that $g:\left(\mathbf{R}^{n}, 0\right) \rightarrow(\mathbf{R}, 0)$ is an analytic function germ, that $I$ is the ideal generated by $f_{1}, \ldots, f_{k}$ and all $(k+1) \times(k+1)$ minors $\frac{\partial\left(g, f_{1}, \ldots, f_{k}\right)}{\partial\left(x_{i_{1}}, \ldots, x_{i_{k+1}}\right)}$ in $\mathcal{O}_{\mathbf{R}^{n}, 0}$ and that $\operatorname{dim}_{\mathbf{R}} \mathcal{O}_{\mathbf{R}^{n}, 0} / I<+\infty$. Our proof differs from [DLNS] because we shall not use neither fixed point theory nor the Lê-Greuel formula.

\subsection{Characterization of a non-degenerate critical point}

We study the following local situation. Let

$$
\begin{array}{ccc}
f:\left(\mathbf{K}^{n}, p\right) & \rightarrow & \left(\mathbf{K}^{k}, \delta\right) \\
x & \mapsto & \left(f_{1}(x), \ldots, f_{k}(x)\right)
\end{array}
$$

be an analytic germ defined near $p(\mathbf{K}=\mathbf{R}$ or $\mathbf{C}) ; \delta$ is a regular value of $f$ so that $f^{-1}(\delta)$ is an analytic manifold of dimension $n-k$ and $p \in f^{-1}(\delta)$. Let $g: \mathbf{K}^{n} \rightarrow \mathbf{K}$ be defined near $p$. We will find a condition for the point $p$ to be a non-degenerate critical point of $g_{\mid f^{-1}(\delta)}$.

Let

$$
M(x)=\operatorname{det}\left[\frac{\partial f_{i}}{\partial x_{j}}(x)\right]_{1 \leq i, j \leq k} .
$$

For shortness we write $x=\left(x^{\prime}, x^{\prime \prime}\right)=\left(x_{1}, \ldots, x_{k} ; x_{k+1}, \ldots, x_{n}\right)$. We can assume that $M(p) \neq 0$ and apply the implicit function theorem in the neighborhood of $p$. There exists an analytic mapping

$$
\varphi: \mathbf{K}^{n-k} \rightarrow \mathbf{K}^{k}, x^{\prime \prime} \mapsto \varphi\left(x^{\prime \prime}\right)
$$

such that $\varphi\left(p^{\prime \prime}\right)=p^{\prime}$ and $f\left(\varphi\left(x^{\prime \prime}\right), x^{\prime \prime}\right)=f(p)=\delta$. We write

$$
\begin{aligned}
& G\left(x^{\prime \prime}\right)=g\left(\varphi\left(x^{\prime \prime}\right), x^{\prime \prime}\right), \\
& m_{j}(x)=\frac{\partial\left(g, f_{1}, \ldots, f_{k}\right)}{\partial\left(x_{1}, \ldots, x_{k}, x_{j}\right)} \quad \text { for } \quad j \geq k+1 .
\end{aligned}
$$

Let $\mathcal{O}_{\mathbf{K}^{n}, p}$ be the ring of germs of analytic functions defined near $p$. Let $I_{p}$ be the ideal in $\mathcal{O}_{\mathbf{K}^{n}, p}$ generated by all $(k+1) \times(k+1)$ minors $\frac{\partial\left(g, f_{1}, \ldots, f_{k}\right)}{\partial\left(x_{i_{1}}, \ldots, x_{i_{k+1}}\right)}$. Let $C_{p}=V\left(I_{p}\right)$ be the set of the zeros of $I_{p}$. Let $J_{p}$ be the ideal generated by $m_{k+1}, \ldots, m_{n}$. We have the following lemmas: 
Lemma 3.1 The function $g_{\mid f^{-1}(\delta)}$ has a critical point at $p$ if and only if $\frac{\partial G}{\partial x_{k+1}}=\cdots=\frac{\partial G}{\partial x_{n}}=0$.

Proof. It is clear.

Lemma 3.2 The function $g_{\mid f^{-1}(\delta)}$ has a critical point at $p$ if and only if $p \in f^{-1}(\delta) \cap C_{p}$.

Proof. It is clear.

Lemma 3.3 $J_{p}=I_{p}$

Proof. It is proved in [Sz2, p.349].

Remark Szafraniec's proof uses the Lê-Greuel formula. We give a direct proof in the appendix at the end of the paper.

Lemma 3.4 The function $g_{\mid f^{-1}(\delta)}$ has a non-degenerate critical point if and only if $C_{p}$ is a regular complete intersection at $p$ of dimension $k$ and $C_{p}$ intersects $f^{-1}(\delta)$ transversally at $p$.

Proof. Let $A(\theta)$ be the following matrix

$$
A(\theta)=\left[\begin{array}{ccc}
\frac{\partial f_{1}}{\partial x_{1}}(\theta) & \ldots & \frac{\partial f_{1}}{\partial x_{n}}(\theta) \\
\vdots & \ddots & \vdots \\
\frac{\partial f_{k}}{\partial x_{1}}(\theta) & \ldots & \frac{\partial f_{k}}{\partial x_{n}}(\theta) \\
\frac{\partial m_{k+1}}{\partial x_{1}}(\theta) & \ldots & \frac{\partial m_{k+1}}{\partial x_{n}}(\theta) \\
\vdots & \ddots & \vdots \\
\frac{\partial m_{n}}{\partial x_{1}}(\theta) & \ldots & \frac{\partial m_{n}}{\partial x_{n}}(\theta)
\end{array}\right] .
$$

As Szafraniec does in [Sz2, p.349-350], we obtain

$$
\operatorname{det} A(p)=(-1)^{k(n-k)} M(p)^{n-k+1} \operatorname{det}\left[\frac{\partial^{2} G}{\partial x_{i} \partial x_{j}}\left(p^{\prime \prime}\right)\right]_{k+1 \leq i, j \leq n} .
$$

This result allows us to conclude because $g_{\mid f^{-1}(\delta)}$ has a non-degenerate critical point if and only if 


$$
\operatorname{det}\left[\frac{\partial^{2} G}{\partial x_{i} \partial x_{j}}\right]_{k+1 \leq i, j \leq n} \neq 0
$$

\subsection{A Morse approximation}

We return to the real case and, using Morse theory for manifolds with boundary, we relate

$$
\chi\left(f^{-1}(\delta) \cap\{g \geq \alpha\} \cap B_{\varepsilon}\right)+\chi\left(f^{-1}(\delta) \cap\{g \leq \alpha\} \cap B_{\varepsilon}\right) \bmod 2
$$

to the number of critical points of a Morse approximation of $g_{\mid f^{-1}(\delta) \cap B_{\varepsilon}}$.

We need the following result about correct critical points of $g_{\mid f^{-1}(\delta) \cap B_{\varepsilon}}$.

Lemma 3.5 Let $\delta$ be a small regular value so that the manifold with boundary $f^{-1}(\delta) \cap B_{\varepsilon}$ is non-singular. Then

- At all correct critical points of $g_{\mid f^{-1}(\delta) \cap B_{\varepsilon}}$ where $g>0$, the gradient of $g_{\mid F_{\delta}}$ points outwards.

- At all correct critical points of $g_{\mid f^{-1}(\delta) \cap B_{\varepsilon}}$ where $g<0$, the gradient of $g_{\mid F_{\delta}}$ points inwards.

- There are no correct critical points of $g_{\mid f^{-1}(\delta) \cap B_{\varepsilon}}$ with $g=0$.

Proof. We prove the first point. In order to prove the second one it is enough to replace $g$ by $-g$. Let $\omega$ be the euclidian distance function and let

$$
\begin{aligned}
& X=\left\{x \in\left(f^{-1}(0) \backslash\{0\}\right) \cap\{g(x)>0\} \mid \exists \lambda(x) \text { and } \mu(x)\right. \\
& \quad \text { with } \nabla g(x)=\lambda(x) \nabla f(x)+\mu(x) \nabla \omega(x) \text { and } \mu(x)<0\} .
\end{aligned}
$$

It is a subanalytic set. If $0 \in \bar{X}$, we apply the curve selection lemma (cf. $[\mathrm{Mi2}])$. There exists an analytic arc $\gamma:\left[0, \varepsilon_{0}[\rightarrow \bar{X}\right.$ such that $\gamma(0)=0$. Then we have for all $t \in\left[0, \varepsilon_{0}[\right.$

$$
\frac{\partial(g \circ \gamma(t))}{\partial t}=\left\langle\nabla g(\gamma(t)), \gamma^{\prime}(t)\right\rangle
$$

and

$$
\frac{\partial(g \circ \gamma(t))}{\partial t}=\lambda(\gamma(t))\left\langle\nabla f(\gamma(t)), \gamma^{\prime}(t)\right\rangle+\mu(\gamma(t))\left\langle\nabla \omega(\gamma(t)), \gamma^{\prime}(t)\right\rangle .
$$

We have for all $t \in\left[0, \varepsilon_{0}\left[,\left\langle\nabla f(\gamma(t)), \gamma^{\prime}(t)\right\rangle=0\right.\right.$ for $\gamma\left(\left[0, \varepsilon_{0}[) \subset f^{-1}(0)\right.\right.$ and 
since $\left\langle\nabla \omega(\gamma(t)), \gamma^{\prime}(t)\right\rangle \geq 0$ for all $t \in\left[0, \varepsilon_{0}[\right.$, we will have

$$
\text { for all } t \in\left[0, \varepsilon_{0}\left[\frac{\partial(g \circ \gamma(t))}{\partial t} \leq 0 .\right.\right.
$$

The function $g \circ \gamma$ is decreasing and so, for all $t \in\left[0, \varepsilon_{0}[, g \circ \gamma(t) \leq g \circ\right.$ $\gamma(0)=0$. But for all $t \neq 0, g \circ \gamma(t)>0$ so $0 \notin \bar{X}$. We can choose $\varepsilon$ sufficiently small so that in $f^{-1}(0) \backslash\{0\} \cap B_{\varepsilon}$, the gradient of $g_{\mid f^{-1}(0) \backslash\{0\}}$ and the gradient of $\omega_{\mid f^{-1}(0) \backslash\{0\}}$ do not point in opposite directions in $\{g>0\}$. Choosing $\delta$ sufficiently close to 0 , this implies that at all correct critical points of $g_{\mid F_{\delta} \cap\{g>0\}}$, the gradient of $g_{\mid f^{-1}(\delta) \cap B_{\varepsilon}}$ will point outwards.

We prove the third point with the same ideas considering the sets

$$
\begin{aligned}
& Y_{>}=\left\{x \in\left(f^{-1}(0) \backslash\{0\}\right) \cap\{g(x)=0\} \mid \exists \lambda(x) \text { and } \mu(x)\right. \\
& \text { with } \nabla g(x)=\lambda(x) \nabla f(x)+\mu(x) \nabla \omega(x) \text { and } \mu(x)>0\}
\end{aligned}
$$

and

$$
\begin{aligned}
& Y_{<}=\left\{x \in\left(f^{-1}(0) \backslash\{0\}\right) \cap\{g(x)=0\} \mid \exists \lambda(x) \text { and } \mu(x)\right. \\
& \text { with } \nabla g(x)=\lambda(x) \nabla f(x)+\mu(x) \nabla \omega(x) \text { and } \mu(x)<0\}
\end{aligned}
$$

and proving that $0 \notin \overline{Y_{>}}$and that $0 \notin \overline{Y_{<}}$.

Now let $\tilde{g}$ be a perturbation of $g$ such that $\tilde{g}_{\mid f^{-1}(\delta) \cap B_{\varepsilon}}$ is a Morse correct function then we have

Lemma 3.6 Let $\delta$ be a small regular value so that the manifold with boundary $f^{-1}(\delta) \cap B_{\varepsilon}$ is non-singular. Let $\alpha \in \mathbf{R}$ such that $|\alpha| \ll|\delta|$ and $(\delta, \alpha)$ is a regular value of $(f, g)$ then

$$
\chi\left(f^{-1}(\delta) \cap\{g \geq \alpha\} \cap B_{\varepsilon}\right)+\chi\left(f^{-1}(\delta) \cap\{g \leq \alpha\} \cap B_{\varepsilon}\right)
$$

is equal to the number of non-degenerate critical points of $\tilde{g}_{\mid f^{-1}(\delta) \cap B_{\varepsilon}}$ modulo 2 .

Proof. For convenience, we denote $f^{-1}(\delta) \cap B_{\varepsilon}$ by $F_{\delta}$ and $f^{-1}(\delta) \cap\{g * \alpha\}$ by $F_{\delta}(g * \alpha)$ where $* \in\{=, \geq, \leq\}$.

Since $\operatorname{dim}_{\mathbf{R}} \mathcal{O}_{\mathbf{R}^{n}, 0} / I<+\infty, g_{\mid f^{-1}(0) \backslash\{0\} \cap\{\omega \leq \varepsilon\}}$ admits no critical points. This implies that for $\varepsilon$ sufficiently small, the levels of $g$ intersect $f^{-1}(0)$ transversally on $f^{-1}(0) \cap\{\varepsilon / 4 \leq \omega \leq \varepsilon\}$. If we choose $\delta$ such that $|\delta| \ll \varepsilon$, the levels of $g$ will also intersect $f^{-1}(\delta)$ transversally on $f^{-1}(\delta) \cap\{\varepsilon / 4 \leq \omega \leq$ 
$\varepsilon\}$, because transversality is an open property. Thus $g_{\mid f^{-1}(\delta) \cap\{\omega<\varepsilon\}}$ admits its critical points on $f^{-1}(\delta) \cap B_{\varepsilon / 4}$ and the critical points of $g_{\mid f^{-1}(\delta) \cap\{\omega=\varepsilon\}}$ are correct critical points of $g_{\mid F_{\delta}}$, because on $\{\omega=\varepsilon\}$ the levels of $g$ and $f^{-1}(\delta)$ are transversal. Moreover correct critical points of $g_{\mid F_{\delta}}$ where $g>0$ (resp. $g<0$ ) point outwards (resp. inwards) and there are no correct critical points near $\{g=0\}$ by the previous lemma.

We choose $\alpha$ close to 0 such that 0 is the only possible critical value of $g_{\mid F_{\delta}}$ in $[-|\alpha|,|\alpha|]$ and such that all correct critical points lie far from the level $\{g=\alpha\}$. We apply the result of Morse theory to the manifold with boundary $F_{\delta}(g=\alpha)$ (see Theorem 2.5) and we get

$$
\chi\left(F_{\delta}(g \geq \alpha), F_{\delta}(g=\alpha)\right)=n_{+}\left(\tilde{g}_{\alpha}\right)-n_{-}\left(\tilde{g}_{\alpha}\right),
$$

where $n_{+}\left(\tilde{g}_{\alpha}\right)$ (resp. $n_{-}\left(\tilde{g}_{\alpha}\right)$ ) is the number of non-degenerate critical points with even (resp. odd) index of $\tilde{g}_{\mid F_{\delta}}$ lying in $F_{\delta}(g \geq \alpha)$. In the same way, we have

$$
\chi\left(F_{\delta}(g \leq \alpha), F_{\delta}(g=\alpha)\right)=(-1)^{n-k}\left(n_{+}\left(\tilde{g}_{-\alpha}\right)-n_{-}\left(\tilde{g}_{-\alpha}\right)\right),
$$

where $n_{+}\left(\tilde{g}_{-\alpha)}\right.$ (resp. $\left.n_{-}\left(\tilde{g}_{-\alpha}\right)\right)$ is the number of non-degenerate critical points with even (resp. odd) index of $\tilde{g}_{\mid F_{\delta}}$ lying in $F_{\delta}(g \leq \alpha)$. Finally we have

$$
\begin{aligned}
& \chi\left(F_{\delta}(g \geq \alpha)\right)+\chi\left(F_{\delta}(g \leq \alpha)\right) \\
& \quad=n_{+}\left(\tilde{g}_{\alpha}\right)+n_{-}\left(\tilde{g}_{\alpha}\right)+n_{+}\left(\tilde{g}_{-\alpha}\right)+n_{-}\left(\tilde{g}_{-\alpha}\right) \bmod 2
\end{aligned}
$$

hence

$$
\begin{aligned}
& \chi\left(F_{\delta}(g \geq \alpha)\right)+\chi\left(F_{\delta}(g \leq \alpha)\right) \\
& \quad=\text { number of non-degenerate critical points of } \tilde{g}_{\mid F_{\delta}} \bmod 2 .
\end{aligned}
$$

\subsection{Study of $\mathcal{O}_{\mathbf{R}^{n}, 0} / I$}

We relate the dimension of $\mathcal{O}_{\mathbf{R}^{n}, 0} / I$ to the number of non-degenerate critical points of a suitable Morse approximation of $g_{\mid f^{-1}(\delta) \cap B_{\varepsilon}}$. Let $J_{\mathbf{C}}$ be the ideal generated in $\mathcal{O}_{\mathbf{C}^{n}, 0}$ by all the $(k+1) \times(k+1)$ minors $\frac{\partial\left(g, f_{1}, \ldots, f_{k}\right)}{\partial\left(x_{i_{1}}, \ldots, x_{i_{k+1}}\right)}$ and let $I_{\mathbf{C}}=\left(f_{1}, \ldots, f_{k} ; J_{\mathbf{C}}\right)$. Let $C=V\left(J_{\mathbf{C}}\right)$. Saito has proved in [Sa] that $\frac{\mathcal{O}_{\mathbf{C}^{n}, 0}}{J_{\mathbf{C}}}$ is a Cohen-Macaulay ring of dimension $k$ and so $C$ is equidimensional of dimension $k$. A result about multiplicity from Serre (see [Se]) gives the 
following relation

$$
\operatorname{dim}_{\mathbf{C}} \frac{\mathcal{O}_{\mathbf{C}^{n}, 0}}{I_{\mathbf{C}}}=\left(f_{\mathbf{C}}^{-1}(0) ; C\right)_{0}=\gamma
$$

where $\left(f_{\mathbf{C}}^{-1}(0) ; C\right)_{0}$ is the intersection multiplicity of $f_{\mathbf{C}}^{-1}(0)$ and $C$ at 0 . Replacing $g$ by a suitable perturbation if necessary, we can assume that $C$ and $f_{\mathbf{C}}^{-1}(\delta)$ intersect transversally at regular points. We have

Lemma 3.7 The function $g_{\mid f^{-1}(\delta) \cap B_{\varepsilon}}$ is a Morse function and $\operatorname{dim}_{\mathbf{R}} \mathcal{O}_{\mathbf{R}^{n}, 0} / I$ is equal to the number of non-degenerate critical points of $g_{\mid f^{-1}(\delta) \cap B_{\varepsilon}}$ modulo 2 .

Proof. Write

$$
C \cap f_{\mathbf{C}}^{-1}(\delta)=\left\{p_{1}, \ldots, p_{r}\right\} \cup\left\{p_{r+1}, \overline{p_{r+1}}, \ldots, p_{m}, \overline{p_{m}}\right\}
$$

where $p_{1}, \ldots, p_{r}$ are real points. Hence

$$
\gamma=\sharp C \cap f_{\mathbf{C}}^{-1}(\delta)=\sharp\left\{p_{1}, \ldots, p_{r}\right\} \bmod 2 .
$$

One can choose $\delta$ sufficiently small so that $\left\{p_{1}, \ldots, p_{r}\right\} \subset B_{\varepsilon}$. Let us examine the situation at $p_{i}$ for $1 \leq i \leq r$. Since $f^{-1}(\delta) \cap B_{\varepsilon}$ is regular, we can assume that, for instance, $M\left(p_{i}\right) \neq 0$ with $M(x)=\operatorname{det}\left[\frac{\partial f_{i}}{\partial x_{j}}(x)\right]_{1 \leq i, j \leq k}$. Using Lemma 3.3 and its proof, we have

$$
\operatorname{det}\left[\begin{array}{ccc}
\frac{\partial f_{1}}{\partial x_{1}}(\theta) & \ldots & \frac{\partial f_{1}}{\partial x_{n}}(\theta) \\
\vdots & \ddots & \vdots \\
\frac{\partial f_{k}}{\partial x_{1}}(\theta) & \ldots & \frac{\partial f_{k}}{\partial x_{n}}(\theta) \\
\frac{\partial m_{k+1}}{\partial x_{1}}(\theta) & \ldots & \frac{\partial m_{k+1}}{\partial x_{n}}(\theta) \\
\vdots & \ddots & \vdots \\
\frac{\partial m_{n}}{\partial x_{1}}(\theta) & \ldots & \frac{\partial m_{n}}{\partial x_{n}}(\theta)
\end{array}\right] \neq 0
$$

because $C$ and $f_{\mathbf{C}}^{-1}(\delta)$ intersect transversally. From this we deduce that $C \cap \mathbf{R}^{n}$, which is the set of real points of $C$, and $f^{-1}(\delta) \cap B_{\varepsilon}$ intersect transversally because all the coefficients involved in the above determinant are real and $p_{i}$ is a real point. Finally we get that $g_{\mid F_{\delta}}$ is a Morse function 
and $p_{1}, \ldots, p_{r}$ are its non-degenerate critical points hence

$$
\operatorname{dim}_{\mathbf{R}} \frac{\mathcal{O}_{\mathbf{R}^{n}, 0}}{I} \equiv r \bmod 2
$$

since $\operatorname{dim}_{\mathbf{R}} \frac{\mathcal{O}_{\mathbf{R}^{n}, 0}}{I}=\operatorname{dim}_{\mathbf{C}} \frac{\mathcal{O}_{\mathbf{C}^{n}, 0}}{I_{\mathbf{C}}}$.

\subsection{Main result and some corollaries}

Now we are ready to state our main theorem.

Theorem 3.8 Let $\delta \in \mathbf{R}^{k}$ be regular value of $f$ and let $\alpha \in \mathbf{R}$ such that $|\alpha| \ll|\delta|$ and $(\delta, \alpha)$ is a regular value of $(f, g)$. If $\operatorname{dim}_{\mathbf{R}} \mathcal{O}_{\mathbf{R}^{n}, 0} / I<+\infty$ then

$$
\begin{aligned}
& \chi\left(f^{-1}(\delta) \cap\{g \geq \alpha\} \cap B_{\varepsilon}\right)+\chi\left(f^{-1}(\delta) \cap\{g \leq \alpha\} \cap B_{\varepsilon}\right) \\
& \quad \equiv \operatorname{dim}_{\mathbf{R}} \frac{\mathcal{O}_{\mathbf{R}^{n}, 0}}{I} \bmod 2 .
\end{aligned}
$$

Proof. It is a combination of Lemma 3.6 and Lemma 3.7.

Corollary 3.9 Let $\delta \in \mathbf{R}^{k}$ be regular value of $f$ and let $\alpha \in \mathbf{R}$ such that $|\alpha| \ll|\delta|$ and $(\delta, \alpha)$ is a regular value of $(f, g)$. If $\operatorname{dim}_{\mathbf{R}} \mathcal{O}_{\mathbf{R}^{n}, 0} / I<+\infty$ then:

$$
\begin{aligned}
& \chi\left(f^{-1}(\delta) \cap B_{\varepsilon}\right)+\chi\left(f^{-1}(\delta) \cap\{g=\alpha\} \cap B_{\varepsilon}\right) \\
& \quad \equiv \operatorname{dim}_{\mathbf{R}} \frac{\mathcal{O}_{\mathbf{R}^{n}, 0}}{I} \bmod 2 .
\end{aligned}
$$

Proof. Using the Mayer-Vietoris sequence, we find

$$
\begin{aligned}
\chi\left(f^{-1}(\delta) \cap B_{\varepsilon}\right)= & \chi\left(f^{-1}(\delta) \cap\{g \geq \alpha\} \cap B_{\varepsilon}\right) \\
& +\chi\left(f^{-1}(\delta) \cap\{g \leq \alpha\} \cap B_{\varepsilon}\right) \\
& -\chi\left(f^{-1}(\delta) \cap\{g=\alpha\} \cap B_{\varepsilon}\right)
\end{aligned}
$$

and it is easy to conclude.

Let $L_{f}$ and $L_{(f, g)}$ be the respective links of $f$ and $(f, g)$. We define, following Wall's notation

$$
\begin{aligned}
\psi(f) & =\frac{1}{2} \operatorname{dim}_{\mathbf{Z}_{2}}\left(H_{*}\left(L_{f}, \mathbf{Z}_{2}\right)\right), \\
\psi((f, g)) & =\frac{1}{2} \operatorname{dim}_{\mathbf{Z}_{2}}\left(H_{*}\left(L_{(f, g)}, \mathbf{Z}_{2}\right)\right) .
\end{aligned}
$$


We have

\section{Corollary 3.10}

$$
\psi(f)+\psi((f, g)) \equiv \operatorname{dim}_{\mathbf{R}} \frac{\mathcal{O}_{\mathbf{R}^{n}, 0}}{I} \bmod 2 .
$$

Proof. We have $L_{f}=f^{-1}(0) \cap S_{\varepsilon}$. For $\delta$ sufficiently small, $L_{f}$ is diffeomorphic to $f^{-1}(\delta) \cap S_{\varepsilon}$. Now it is enough to apply the following relation

$$
\frac{1}{2} \operatorname{dim}_{\mathbf{Z}_{2}}\left(H_{*}\left(f^{-1}(\delta) \cap S_{\varepsilon}, \mathbf{Z}_{2}\right)\right) \equiv \chi\left(f^{-1}(\delta) \cap S_{\varepsilon}\right) \bmod 2,
$$

which is well-known if $n-k$ is odd and which is proved, for instance, in [ASV] Proposition 3.4, if $n-k$ is even.

\section{Topological invariance of the Milnor number $\bmod 2$}

We give here an alternative proof of the topological invariance of the Milnor number mod 2 proved in [Wa]. Our proof does not use the methods of fixed point theory.

Let $f=\left(f_{1}, \ldots, f_{k}\right):\left(\mathbf{R}^{n}, 0\right) \rightarrow\left(\mathbf{R}^{k}, 0\right)$, with $n>k$, be an analytic germ of finite singularity type and let $f_{\mathbf{C}}$ be the complexification of $f$. Then according to Wall [Wa], $f_{\mathbf{C}}$ has an isolated singularity and we can define its Milnor number $\mu\left(f_{\mathbf{C}}\right)$. We define the Milnor number of $f$ by $\mu(f)=\mu\left(f_{\mathbf{C}}\right)$ and we will prove that

$$
\psi(f)=1+\mu(f) \bmod 2,
$$

where $\psi(f)$ is defined as in the previous section.

We need the following version of Sard's lemma.

Lemma 4.1 Let $N \subset M \subset \mathbf{R}^{N}$ be analytic sets and let $N_{\mathbf{C}}$ and $M_{\mathbf{C}}$ be their respective complexifications. Assume that $M_{\mathbf{C}} \backslash N_{\mathbf{C}}$ is a smooth complex manifold of dimension $K$. Let $\pi: \mathbf{R}^{N} \rightarrow \mathbf{R}^{P}$, with $P \leq K$, be an analytic mapping and let $\pi_{\mathbf{C}}$ be its complexification. Then for almost all $\beta \in \mathbf{R}^{P}, \pi_{\mathbf{C}}^{-1}(\beta) \cap M_{\mathbf{C}} \backslash N_{\mathbf{C}}$ is a smooth manifold of dimension $K-P$.

Proof. Let $\Sigma_{\mathbf{C}}$ be the critical set of $\pi_{\mathbf{C} \mid M_{\mathbf{C}} \backslash N_{\mathbf{C}}}$ and let $\Sigma$ be the critical set of $\pi_{\mid M \backslash N}$. Then $\pi_{\mathbf{C}}\left(\Sigma_{\mathbf{C}}\right)$ has at most dimension $P-1$ and $\pi(\Sigma) \subset \pi_{\mathbf{C}}\left(\Sigma_{\mathbf{C}}\right) \cap$ $\mathbf{R}^{P}$ is a subanalytic set of dimension at most $P-1$, so for $\beta \in \mathbf{R}^{P} \backslash \pi(\Sigma)$, $\beta \notin \pi_{\mathbf{C}}\left(\Sigma_{\mathbf{C}}\right)$ which means that $\beta$ is a regular value of $\pi_{\mathbf{C}}: M_{\mathbf{C}} \backslash N_{\mathbf{C}} \rightarrow \mathbf{C}^{P}$. 
Lemma 4.2 Let $f=\left(f_{1}, \ldots, f_{k}\right):\left(\mathbf{R}^{n}, 0\right) \rightarrow\left(\mathbf{R}^{k}, 0\right)$, with $n>k$, be an analytic germ of finite singularity type. There exists an analytic germ $g:\left(\mathbf{R}^{n}, 0\right) \rightarrow(\mathbf{R}, 0)$ such that $F_{\mathbf{C}}=\left(f_{\mathbf{C}}, g_{\mathbf{C}}\right):\left(\mathbf{R}^{n}, 0\right) \rightarrow\left(\mathbf{R}^{k+1}, 0\right)$ has an isolated singularity or, equivalently, $(f, g)$ is of finite singularity type.

Proof. Consider the following analytic map

$$
\begin{array}{ccc}
H:\left(\mathbf{R}^{n} \times \mathbf{R}^{n},(0,0)\right) & \rightarrow & \left(\mathbf{R}^{k+1}, 0\right) \\
(x, a) & \mapsto & \left(f_{1}, \ldots, f_{k}, a_{1} x_{1}+\cdots+a_{n} x_{n}\right) .
\end{array}
$$

The derivative of $H$ at $(x, a)$ is given by the following matrix

$$
D H(x, a)=\left(\begin{array}{cccccc}
\frac{\partial f_{1}}{\partial x_{1}} & \cdots & \frac{\partial f_{1}}{\partial x_{n}} & 0 & \cdots & 0 \\
\vdots & \ddots & \vdots & \vdots & \ddots & \vdots \\
\frac{\partial f_{k}}{\partial x_{1}} & \cdots & \frac{\partial f_{k}}{\partial x_{n}} & 0 & \cdots & 0 \\
a_{1} & \cdots & a_{n} & x_{1} & \cdots & x_{n}
\end{array}\right)
$$

If $(x, a) \in H^{-1}(0)$ and $x \neq 0$ then there exists $i \in\{1, \ldots, n\}$ with $x_{i} \neq 0$ and then rank $D H(x, a)=k+1$ since

$$
\operatorname{rank}\left(\begin{array}{cccc}
\frac{\partial f_{1}}{\partial x_{1}} & \cdots & \frac{\partial f_{1}}{\partial x_{n}} & 0 \\
\vdots & \ddots & \vdots & \vdots \\
\frac{\partial f_{k}}{\partial x_{1}} & \cdots & \frac{\partial f_{k}}{\partial x_{n}} & 0 \\
a_{1} & \cdots & a_{n} & x_{i}
\end{array}\right)=k+1
$$

So $X=H^{-1}(0) \backslash\left(\{0\} \times \mathbf{R}^{n}\right)$ is an analytic manifold of dimension $2 n-(k+$ 1). Consider

$$
\begin{array}{rlc}
\pi: H^{-1}(0) & \rightarrow & \left(\mathbf{R}^{n}, 0\right) \\
(x, a) & \mapsto & a
\end{array}
$$

the projection map on the second component. Using the above lemma, we can find $a \in \mathbf{R}^{n}$ such that $X_{\mathbf{C}} \cap \pi_{\mathbf{C}}^{-1}(a)$ is a smooth analytic manifold of dimension $n-(k+1)$, where $X_{\mathbf{C}}=H_{\mathbf{C}}^{-1}(0) \backslash\left(\{0\} \times \mathbf{C}^{n}\right)$ and $\pi_{\mathbf{C}}$ is the complexification of $\pi$. This exactly means that $\left(f_{\mathbf{C}}, a_{1} x_{1}+\cdots+a_{n} x_{n}\right)$ : $\left(\mathbf{C}^{n}, 0\right) \rightarrow\left(\mathbf{C}^{k+1}, 0\right)$ has an isolated singularity. 
Lemma 4.3 Let $f=\left(f_{1}, \ldots, f_{k}\right):\left(\mathbf{R}^{n}, 0\right) \rightarrow\left(\mathbf{R}^{k}, 0\right)$, with $n>k$, be an analytic germ of finite singularity type and let $g:\left(\mathbf{R}^{n}, 0\right) \rightarrow(\mathbf{R}, 0)$ be an analytic germ such that $F_{\mathbf{C}}=\left(f_{\mathbf{C}}, g_{\mathbf{C}}\right):\left(\mathbf{R}^{n}, 0\right) \rightarrow\left(\mathbf{R}^{k+1}, 0\right)$ has an isolated singularity then

$$
\operatorname{dim}_{\mathbf{R}} \frac{\mathcal{O}_{\mathbf{R}^{n}, 0}}{\left(f_{1}, \ldots, f_{k}, \frac{\partial\left(g, f_{1}, \ldots, f_{k}\right)}{\partial\left(x_{i_{1}}, \ldots, x_{i_{k+1}}\right)}\right)}<+\infty
$$

Proof. Suppose that this vector space is not finite dimensional. Then $0 \in$ $\mathbf{C}^{n}$ is not isolated in

$$
f_{\mathbf{C}}^{-1}(0) \cap\left(\cap_{1 \leq i_{1}<\cdots<i_{k+1} \leq n}\left\{\frac{\partial\left(g_{\mathbf{C}}, f_{1 \mathbf{C}}, \ldots, f_{k \mathbf{C}}\right)}{\partial\left(x_{i_{1}}, \ldots, x_{i_{k+1}}\right)}=0\right\}\right) .
$$

Hence, by the curve selection lemma $([\mathrm{Mi} 2])$, there exists a real analytic curve $P(t):[0, \lambda) \rightarrow \mathbf{C}^{n}$ such that $P(0)=0, f_{\mathbf{C}}(P(t))=0$ and $\frac{\partial\left(g_{\mathbf{C}}, f_{1 \mathbf{C}}, \ldots, f_{k \mathbf{C}}\right)}{\partial\left(x_{i_{1}}, \ldots, x_{i_{k+1}}\right)}(P(t))=0$ for all $(k+1)$-tuple $\left(i_{1}, \ldots, i_{k+1}\right)$. This means that the vectors $\nabla f_{j \mathbf{C}}, j=1, \ldots, k$ and $\nabla g_{\mathbf{C}}$ are linearly dependent over $\mathbf{C}$ at $P(t)$ and there exist $a_{1}(t), \ldots, a_{k}(t)$ and $b(t)$ such that

$$
\sum_{j=1}^{k} a_{j}(t) \cdot \nabla f_{j_{\mathbf{C}}}(P(t))=b(t) \cdot \nabla g_{\mathbf{C}}(P(t)),
$$

and $\left(a_{1}(t), \ldots, a_{n}(t), b(t)\right) \neq(0, \ldots, 0)$. Since $f$ is of finite singularity type, $f_{\mathbf{C}}^{-1}(0)$ is a smooth $(n-k)$-dimensional complex manifold outside the origin and $\nabla f_{1 \mathbf{C}}(P(t)), \ldots, \nabla f_{k \mathbf{C}}(P(t))$ are linearly independent for $t \neq 0$. This implies that for all $t \neq 0, b(t) \neq 0$ and

$$
\overline{\nabla\left(g_{\mathbf{C}}\right)(P(t))}=\sum_{j=1}^{k} \overline{c_{j}(t)} \cdot \overline{\nabla f_{j \mathbf{C}}(P(t))},
$$

where $c_{j}(t)=\frac{a_{j}(t)}{b(t)}$. Therefore

$$
\begin{aligned}
& \frac{\partial}{\partial t}(g \circ P(t))=\left\langle\frac{\partial P(t)}{\partial t}, \overline{\nabla\left(g_{\mathbf{C}}\right)(P(t))}\right\rangle \\
& \frac{\partial}{\partial t}(g \circ P(t))=\left\langle\frac{\partial P(t)}{\partial t}, \sum_{j=1}^{k} \overline{c_{j}(t)} \cdot \overline{\nabla f_{j \mathbf{C}}(P(t))}\right\rangle
\end{aligned}
$$




$$
=\sum_{j=1}^{k} c_{j}(t) \cdot \frac{\partial}{\partial t}\left(f_{j} \circ P(t)\right)=0
$$

since $\{P(t)\} \subset f_{\mathbf{C}}^{-1}(0)$. Thus we have $\{P(t)\} \subset\{g=0\}$ which contradicts the fact that $\left(f_{\mathbf{C}}, g_{\mathbf{C}}\right)$ has an isolated singularity.

Before proving our main result, we need to prove it in the case of curves.

Lemma 4.4 Let $F:\left(\mathbf{R}^{n}, 0\right) \rightarrow\left(\mathbf{R}^{n-1}, 0\right)$ be a complete intersection of finite singularity type. Then

$$
\psi(F) \equiv 1+\mu(F) \bmod 2 \text {. }
$$

Proof. We have

$$
\mu=2 \delta-r+1
$$

where $r$ is the number of branches of the complex curve $F_{\mathbf{C}}^{-1}(0)$ (see $[\mathrm{BuG}$, Mi2]). The complex conjugation acts on the set of branches: those interchanged in pairs do not yield a real branch, whereas those left invariant yield a single real branch. Thus $r \equiv s \bmod 2$ where $s$ is the number of real branches of $f^{-1}(0)$. Now we just have to use the fact that

$$
s \equiv \frac{1}{2} \chi\left(F^{-1}(0) \cap S_{\varepsilon}\right) \equiv \psi(F) \bmod 2 .
$$

Now we are ready to state:

Theorem 4.5 Let $f:\left(\mathbf{R}^{n}, 0\right) \rightarrow\left(\mathbf{R}^{k}, 0\right)$ be an analytic germ of finite singularity type then

$$
\psi(f) \equiv 1+\mu(f) \bmod 2
$$

Proof. With Lemma 4.2 we construct $n-k-1$ functions $g_{1}, \ldots, g_{n-k-1}$ such that the $n-k-1$ mappings

$$
\begin{array}{cccc}
F_{1}=\left(f, g_{1}\right) & :\left(\mathbf{R}^{n}, 0\right) & \rightarrow & \left(\mathbf{R}^{k+1}, 0\right), \\
F_{2}=\left(f, g_{1}, g_{2}\right) & :\left(\mathbf{R}^{n}, 0\right) & \rightarrow & \left(\mathbf{R}^{k+2}, 0\right), \\
\vdots & \vdots \\
F_{n-k-1}=\left(f, g_{1}, \ldots, g_{n-k-1}\right) & :\left(\mathbf{R}^{n}, 0\right) & \rightarrow\left(\mathbf{R}^{n-1}, 0\right),
\end{array}
$$


are of finite singularity type. Applying Corollary 3.10, Lemma 4.3 and the Lê-Greuel formula, we find

$$
\begin{array}{clc}
\psi(f)+\psi\left(F_{1}\right) & \equiv & \mu(f)+\mu\left(F_{1}\right) \bmod 2 \\
\psi\left(F_{1}\right)+\psi\left(F_{2}\right) & \equiv & \mu\left(F_{1}\right)+\mu\left(F_{2}\right) \bmod 2 \\
\vdots & & \vdots \\
\psi\left(F_{n-k-2}\right)+\psi\left(F_{n-k-1}\right) & \equiv & \mu\left(F_{n-k-2}\right)+\mu\left(F_{n-k-1}\right) \bmod 2 .
\end{array}
$$

Adding them together leads to

$$
\psi(f)+\psi\left(F_{n-k-1}\right) \equiv \mu(f)+\mu\left(F_{n-k-1}\right) \bmod 2 .
$$

By the previous lemma, we know that $\psi\left(F_{n-k-1}\right) \equiv 1+\mu\left(F_{n-k-1}\right) \bmod 2$ and it is easy to see that

$$
\psi(f) \equiv 1+\mu(f) \bmod 2 .
$$

\section{Appendix : a direct proof of Lemma 3.3}

We give here a direct, but rather technical, proof of Lemma 3.3. We are in the following situation:

$$
\begin{array}{ccc}
f:\left(\mathbf{K}^{n}, p\right) & \rightarrow & \left(\mathbf{K}^{k}, \delta\right) \\
x & \mapsto & \left(f_{1}(x), \ldots, f_{k}(x)\right),
\end{array}
$$

where $\delta$ is a regular value of $f$ so that $f^{-1}(\delta)$ is an analytic manifold of dimension $n-k$. Let

$$
M(x)=\operatorname{det}\left[\frac{\partial f_{i}}{\partial x_{j}}(x)\right]_{1 \leq i, j \leq k} .
$$

We adopt the notation $f_{i_{x_{j}}}$ for $\frac{\partial f_{i}}{\partial x_{j}}$. We assume that $M(p) \neq 0$ which implies that $\frac{1}{M}$ is analytic at $p$ and that for all $x$ near $p, M(x) \neq 0$. We have for all $j \in\{1, \ldots, n\}$

$$
m_{j}(x)=\left|\begin{array}{cccc}
g_{x_{1}}(x) & \cdots & g_{x_{k}}(x) & g_{x_{j}}(x) \\
f_{1_{x_{1}}}(x) & \cdots & f_{1_{x_{k}}}(x) & f_{1_{x_{j}}}(x) \\
\vdots & \ddots & \vdots & \vdots \\
f_{k_{x_{1}}}(x) & \cdots & f_{k_{x_{k}}}(x) & f_{k_{x_{j}}}(x)
\end{array}\right|
$$


hence, developing $m_{j}$ along the first line and since $M \neq 0$, and omitting the $x$

$$
g_{x_{j}}=\frac{(-1)^{k}}{M}\left(m_{j}+\sum_{i=1}^{k}(-1)^{i} g_{x_{i}} \frac{\partial\left(f_{1}, \ldots, f_{k}\right)}{\partial\left(x_{1}, \ldots, \hat{x}_{i}, \ldots, x_{k} ; x_{j}\right)}\right) .
$$

Remark For all $1 \leq j \leq k \quad m_{j}=0$.

We have for all $\left(i_{1}, \ldots, i_{k+1}\right)$

$$
\frac{\partial\left(g, f_{1}, \ldots, f_{k}\right)}{\partial\left(x_{i_{1}}, \ldots, x_{i_{k+1}}\right)}=\left|\begin{array}{ccc}
g_{x_{i_{1}}} & \cdots & g_{x_{i_{k+1}}} \\
f_{1_{x_{1}}} & \cdots & f_{1_{x_{i_{k+1}}}} \\
\vdots & \ddots & \vdots \\
f_{k_{x_{i_{1}}}} & \cdots & f_{k_{x_{i_{k+1}}}}
\end{array}\right|
$$

We develop along the first line and get

$$
\frac{\partial\left(g, f_{1}, \ldots, f_{k}\right)}{\partial\left(x_{i_{1}}, \ldots, x_{i_{k+1}}\right)}=\sum_{l=1}^{k+1}(-1)^{l+1} g_{x_{i_{l}}} \frac{\partial\left(f_{1}, \ldots, f_{k}\right)}{\partial\left(x_{i_{1}}, \ldots, \hat{i_{l}}, \ldots, x_{i_{k+1}}\right)}
$$

Replacing $g_{x_{i_{l}}}$ by the expression (1), we obtain

$$
\begin{aligned}
& \frac{\partial\left(g, f_{1}, \ldots, f_{k}\right)}{\partial\left(x_{i_{1}}, \ldots, x_{i_{k+1}}\right)} \\
& =\sum_{l=1}^{k+1} \frac{(-1)^{k+l+1}}{M} \frac{\partial\left(f_{1}, \ldots, f_{k}\right)}{\partial\left(x_{i_{1}}, \ldots, \hat{x_{l}}, \ldots, x_{i_{k+1}}\right)} m_{i_{l}}+\sum_{i=1}^{k} \frac{(-1)^{k+1} g_{x_{i_{l}}}}{M} \\
& \quad\left(\sum_{l=1}^{k+1}(-1)^{l+1} \frac{\partial\left(f_{1}, \ldots, f_{k}\right)}{\partial\left(x_{1}, \ldots, \hat{x_{i}}, \ldots, x_{k} ; x_{i_{l}}\right)} \frac{\partial\left(f_{1}, \ldots, f_{k}\right)}{\partial\left(x_{i_{1}}, \ldots, \hat{x_{l}}, \ldots, x_{i_{k+1}}\right)}\right) .
\end{aligned}
$$

It is enough to prove that each term between the big parenthesis is zero. But one sees clearly that is the determinant of the following matrix

$$
B=\left[\begin{array}{ccc}
\frac{\partial\left(f_{1}, \ldots, f_{k}\right)}{\partial\left(x_{1}, \ldots, \hat{x}_{i}, \ldots, x_{k} ; x_{i_{1}}\right)} & \cdots & \frac{\partial\left(f_{1}, \ldots, f_{k}\right)}{\partial\left(x_{1}, \ldots, \hat{x}_{i}, \ldots, x_{k} ; x_{i_{k+1}}\right)} \\
\frac{\partial f_{1}}{\partial x_{i_{1}}} & \cdots & \frac{\partial f_{1}}{\partial x_{i_{k+1}}} \\
\vdots & \ddots & \vdots \\
\frac{\partial f_{k}}{\partial x_{i_{1}}} & \cdots & \frac{\partial f_{k}}{\partial x_{i_{k+1}}}
\end{array}\right]
$$


But we have for all $l \in\{1, \ldots, k+1\}$,

$$
\frac{\partial\left(f_{1}, \ldots, f_{k}\right)}{\partial\left(x_{1}, \ldots, \hat{x}_{i}, \ldots, x_{k} ; x_{i_{l}}\right)}=\sum_{j=1}^{k}(-1)^{j+k} \frac{\partial f_{j}}{\partial x_{i_{l}}} A_{j}
$$

where

$$
A_{j}=\frac{\partial\left(f_{1}, \ldots, \hat{f}_{j}, \ldots, f_{k}\right)}{\partial\left(x_{1}, \ldots, \hat{x}_{i}, \ldots, x_{k}\right)}
$$

and then

$$
L_{0}=\sum_{j=1}^{k}(-1)^{j+k} A_{j} L_{j}
$$

where $L_{0}, L_{1}, \ldots, L_{k}$ are the lines of the matrix $B$, which allows us to conclude that $\operatorname{det} B=0$ and $\frac{\partial\left(g, f_{1}, \ldots, f_{k}\right)}{\partial\left(x_{i_{1}}, \ldots, x_{i_{k}}\right)}$ belongs to the ideal generated by the $m_{i_{l}}$, which is included in $J_{p}$.

\section{References}

[AFS] Aoki K., Fukuda T. and Sun W.Z., On the number of branches of a plane curve germ. Kodai Math. Journal 9 (1986), 179-187.

[AFN1] Aoki K., Fukuda T. and Nishimura T., On the number of branches of the zero locus of a map germ $\left(\mathbf{R}^{n}, 0\right) \rightarrow\left(\mathbf{R}^{n-1}, 0\right)$. Topology and Computer Science: Proceedings of the Symposium held in honor of S. Kinoshita, H. Noguchi and T. Homma on the occasion of their sixtieth birhtdays, (1987), 347-363.

[AFN2] Aoki K., Fukuda T. and Nishimura T., An algebraic formula for the topological types of one parameter bifurcations diagrams. Archive for Rational Mechanics and Analysis 108 (1989), 247-265.

[Ar] Arnold V.I., Index of a singular point of a vector field, the Petrovski-Oleinik inequality, and mixed Hodge structures. Funct. Anal. and its Appli. 12 (1978), $1-14$.

[ASV] Aguilar M.A., Seade J.A. and Verjovsky A., Indices of vector fields and topological invariants of real analytic singularities. J. reine angew. Math. 504 (1998), 159176.

[BuG] Buchweiz R.O. and Greuel G.M., The Milnor number and deformations of complex curves singularities. Invent. Math. 58 (1980), 241-281.

[DLNS] Dudzinski P., Lecki, A., Nowak-Przygodzki P. and Szafraniec Z., On the topological invariance of the Milnor number mod 2. Topology 32 (1993), 573-576.

[Ei] Eisenbud D., An algebraic approach to the topological degree of a smooth map. Bull. Amer. Math. Soc. 84 (1978), 751-764. 
[EL] Eisenbud D. and Levine H.I., An algebraic formula for the degree of a $C^{\infty}$ mapgerm. Annals of Mathematics 106 (1977), 19-44.

[Fu1] Fukui T., An algebraic fomula for a topological invariant of bifurcation of 1parameter family of function-germs. In Stratifications, singularities, and differential equations, II (Marseille, 1990; Honolulu, HI, 1990), Travaux en Cours 55, Hermann, Paris (1997), 45-54.

[Fu2] Fukui T., Mapping degree formula for 2-parameter bifurcation of function-germs. Topology 32 (1993), 567-571.

[Gr] Greuel G.M., Der Gauss-Manin Zusammenhang isolierter Singularitäten von vollständingen Durschnitten. Math. Annalen 214 (1975), 235-266.

[Ha] Hamm H., Lokale topologische Eigenschaften komplexer Raume. Math. Ann. 191 (1971), 235-252.

[HL] Hamm H. and Le Dung Trang, Un théorème de Zariski du type de Lefschetz. Ann. Sci. Ecol. Norm. Sup. (3) 6 (1973), 317-355.

[Kh] Khimshiashvili G.M., On the local degree of a smooth map. Soobshch. Akad. Nauk Gruz. SSR 85 (1977), 309-311.

[Le] Le Dung Trang, Calcul du nombre de Milnor d'une singularité isolée d'intersection complète. Funct. Anal. Appl. 8 (1974), 45-52.

[Mi1] Milnor J., Morse theory. Ann. Math. Stud. 51, Princeton University Press (1963).

[Mi2] Milnor J., Singular points of complex hypersurfaces. Ann. Math. Stud. 61, Princeton University Press (1968).

[Pa] Palamodov V.P., Multiplicity of holomorphic mappings. Funct. Anal. Appl. 1 (1967), 218-226.

[Sa] Saito K., Regularity of Gauss-Manin connection of flat family of isolated singularity. Quelques journées singulières, Publ. du Centre de Math. de l'Ecole Polytechnique (1973).

[Se] Serre J.P., Algèbre locale Multiplicités. Lectures Notes in Math.

[Sz1] Szafraniec Z., On the number of branches of a 1-dimensional semi-analytic set. Kodai Math. Journal 11 (1988), 78-85.

[Sz2] Szafraniec Z., A formula for the Euler characteristic of a real algebraic manifold. Manuscripta mathematica 85 (1994), 345-360.

[Wa] Wall C.T.C., Topological invariance of the Milnor number mod 2. Topology 22 (1983), 345-350.

IMR, Université de Rennes 1

Campus de Beaulieu

35042 Rennes Cedex, France

E-mail: dutertre@maths.univ-rennes1.fr 